\title{
The prognostic significance of HLA-A2 expression on somatic cells in patients with left-sided colon and rectal cancers
}

\author{
Katarzyna Dyląg-Trojanowska', Radosław Pach², Maciej Siedlar³, \\ Jarosław Baran³, Justyna Zybaczyńska4, Antoni M. Szczepanik²
}

${ }^{1}$ Department of General and Oncological Surgery, John Gawlik Hospital, Sucha Beskidzka, Poland

${ }_{2}^{2}$ First Department of General, Oncological and Gastroenterological Surgery, Jagiellonian University Medical College, Krakow, Poland

${ }^{3}$ Department of Clinical Immunology, Institute of Pediatrics, Jagiellonian University Medical College, Krakow, Poland

${ }^{4}$ Penn State Health Hershey Medical Center, Hershey, PA, USA

Introduction. Current knowledge about colorectal cancer (CRC) identifies tumor immunogenicity as one of the more important issues. In cancers, a prerequisite for immune system activation is the presentation of tumor associated antigen (TAA) epitopes to immunocompetent cells. HLA-A2 is one of the antigens in the context of which TAAs are present, but data on the possible impact of HLA-A2 antigen expression on the survival of patients with colorectal cancer are scarce and sometimes contradictory. The aim of this study was to analyse the relationship between HLA-A2 expression in patients with left-sided colorectal cancer in various stages of disease and their long-term survival, and to answer the question of whether a lack of HLA-A2 expression is actually a negative prognostic factor.

Material and methods. A prospective analysis of 58 patients with left-sided colorectal cancer was carried out. Expression of HLA-A2 was determined by patient blood lymphocyte staining, and analysed using flow cytometry.

Results. In the study group, patients with HLA-A2 expression lived statistically longer than HLA-A2 negative patients $(p=0.027)$. There was no significant difference in overall survival between the HLA-A2+ and HLA-A2- groups with stage II and III left-sided CRC. However, the Cox proportional hazard model showed that a lack of HLA-A2 expression was a negative prognostic factor in the group of radically operated patients without distant metastases.

Conclusions. HLA-A2 status may affect the clinical course of patients with left-sided colon and rectal cancer, although left-sided tumors are less immunogenic than right-sided ones. HLA-A2-positive patients with left-sided colorectal cancer lived statistically longer than those who were HLA-A2-negative $(p=0.027)$. Lack of HLA-A2 expression was a negative prognostic factor in the group of radically operated patients.

NOWOTWORY J Oncol 2020; 70, 3: 92-97

Key words: HLA, HLA-A2, left-sided colon cancer, overall survival, prognostic biomarker

\section{Introduction}

The appropriate immune response to tumor cells is dependent on their ability to be recognized by immunocompetent cells. Tumor associated antigens (TAA), or their epitopes, are presented by antigen presenting cells (APCs) in the context of human leukocyte antigen (HLA) expression. SomeTAA-derived

\section{How to cite:}

Dyląg-Trojanowska K, Pach R, Siedlar M, Baran J, Zybaczyńska J, Szczepanik AM. The prognostic significance of HLA-A2 expression on somatic cells in patients with left-sided colon and rectal cancers. NOWOTWORY J Oncol 2020; 70: 92-97. 
epitopes require a specific HLA, or other antigens, for presentation. HLA class I antigens are integral membrane glycoproteins that are inherited and expressed at varying levels on the surface of virtually all somatic cells [1]. It is known that HLA-A2 (MHC class I) is common among Caucasians (approximately $45 \%$ of the Caucasian population) [2] and that some antigens characteristic for colorectal cancer (CRC) are presented in its context. These include CSNK1A1, GAS7, HAUS3, SRPX, WDR46, ERBB2, AKAP13, and MUC1 antigens.

A large proportion of cancer vaccine research has been limited to the HLA-A2-positive population, and HLA-A2-negative patients have been used as a control group in targeted immunotherapy studies [3]. However, some findings show that HLA status itself can influence the clinical course of the disease, as the natural immune response may differ between patients with or without this antigen expression.

Many studies have described HLA expression in patients with cancers of the head and neck [4], breast [5], ovary [6, 7, 8], prostate [8], primary laryngeal squamous cell cancers [9], non-small cell lung cancer [10], melanoma [1], as well as on tumor cells.

Data on the possible impact of HLA-A2 antigen expression on the survival of patients with colorectal cancer are limited and sometimes contradictory. Most data covers a mixed population of right and left-sided colon and rectal cancer patients. Since we know that MHC class I expression is often absent in micro-satellite instability (MSI) tumors [11, 12], which are clinically characterized as having a favorable prognosis and are more frequently observed in right-sided colon tumors [13, 14], the results obtained from a mixed population of all colon and rectal cancer patients might not be representative [15].

According to UICC cancer statistics published in 2018, colorectal cancer is the $3^{\text {rd }}$ cancer in terms of incidence and second in cancer-related mortality in the world. Despite progress in prevention and therapy, there is still space for new therapies and translational research in colorectal cancer. In recent years, several trials on immunotherapy using checkpoint inhibitors in colorectal cancer have produced promising results [16]. However, more than $50-80 \%$ of cancer patients fail to respond to checkpoint inhibitor therapy [17]. Therefore, the investigation of predictive and prognostic factors in various subgroups of colorectal cancer patients is justified.

Colorectal cancer is not a homogenous disease in terms of primary tumor location, and there is evidence that right-sided and left-sided cancers may have different biologies and prognoses [18-21]. CRC immunogenicity, understood as the ability to induce an immune response, also differs between the right and left sides of the colon. There are two different types of colorectal cancer - those which are highly immunogenic (with multiple DNA mutations, chromosomal stability (CS), microsatellite instability (MSI) phenotype and the presence of multiple tumor-infiltrating lymphocytes (TILs), prevalent in the right colon) and those with low immunogenicity (with a limited number of DNA mutations, a microsatellite stable (MSS) and chromosomally instable (CIN) phenotype located in the left side of the colon and rectum) [22]. This heterogeneity should be used to stratify patients in order to provide them with the most optimal, current, and novel immune-based therapeutic strategies available in clinical practice [21].

In this study, we concentrated on tumors located in the left colon and rectum - a more homogenous sub-group of CRC. Locally advanced malignancies were the main area of interest.

The aim of this study was to answer the question of whether HLA-A2 expression is an important prognostic factor in left-sided CRC, which may present with decreased immunogenicity compared to right-sided CRC.

\section{Material and methods}

The study group consisted of 58 colorectal cancer patients treated in a single institution between 2007 and 2012. Only patients with tumors located in the rectum or left colon were included. The term left colon was defined as the large intestine, from the left $1 / 3$ of the transverse colon distally. All patients had histologically confirmed disease, were over 18 years-old, and had had an electively performed surgical procedure. Patients with simultaneous right-sided colon cancer or patients with a history of other neoplastic diseases were excluded. Preoperative radiotherapy was used in 3 of the rectal cancer patients. All patients had no history of autoimmune diseases or recent infections. The group was composed of 25 women and 33 men, with a mean age of 66 (SD 11), in varying stages of disease (tab. I).

The surgical procedures were carried out according to oncological guidelines. Due to the changes of the TNM staging systems during the study period, all the specimens were re-staged according to the $7^{\text {th }}$ edition of the TNM. The clinical and pathological data were recorded. Patients received postoperative chemotherapy if indicated. All patients were followed up for at least 5 years, or until death, and dates of death were verified by the census registry office.

All patients provided their informed, written consent. The study was approved by the Jagiellonian University Ethical Committee KBET no. 86/B/2007 and KBET no. 122.6120.128.2015. The study was registered at ClinicalTrials.gov, registration number NCT03640572.

Blood samples were collected prior to any interventional procedure in sterile EDTA vacutainers. Cell preparation was started 1-2 hours after a blood draw. Expression of HLA-A2 was determined by patient blood lymphocyte staining, using PE-conjugated mouse anti-human HLA-A2 mAb or PE-conjugated isotype-matched mouse immunoglobulins (both BD Pharmingen) as a negative control, followed by lysis of erythrocytes (FACS Lysing Solution, BD Biosciences) and flow cytometry analysis (FACS Canto).

\section{Statistics}

The statistical analysis was conducted using the software Statistica 13 (StatSoft Inc.). The Kaplan-Meier method was used for the calculation of survival probabilities and the Wilcoxon- 
Table I. Clinicopathological characteristics of the patients

\begin{tabular}{|c|c|}
\hline Tumor location & Number of patients \\
\hline Left colon & 27 \\
\hline Rectum & 31 \\
\hline $\mathrm{T} 1$ & 0 \\
\hline T2 & 9 \\
\hline T3 & 39 \\
\hline T4 & 10 \\
\hline NO & 25 \\
\hline N1 & 15 \\
\hline N2 & 14 \\
\hline $\mathrm{Nx}$ & 4 \\
\hline MO & 46 \\
\hline M1 & 12 \\
\hline Stage I & 7 \\
\hline Stage II & 18 \\
\hline Stage III & 21 \\
\hline Stage IV & 12 \\
\hline Grade 1 & 17 \\
\hline Grade 2 & 25 \\
\hline Grade 3 & 9 \\
\hline Grade not assessed & 7 \\
\hline Ro & 44 \\
\hline R1 & 2 \\
\hline R2 & 12 \\
\hline
\end{tabular}

-Gehan test for the comparison of survival curves between groups. The Cox proportional hazard model was used for multivariate analysis. The Chi-square test and the Fisher exact test were used to compare the clinical and pathological features between the HLA-A2-positive and negative groups. Mean patient age was compared using Mann Whitney's U test. A p-value of $<0.05$ was established as statistically significant.

\section{Results}

Fifty-eight colorectal cancer patients were evaluated; 33 were found to be HLA-A2-positive (56\% of whole study group). The distribution of clinicopathological characteristics of patients in HLA-A2+ and HLA-A2- groups are listed in table II.
Table II. Clinicopathological characteristics of patients in HLAA2+ and HLAA2- groups

\begin{tabular}{|c|c|c|c|}
\hline & $\begin{array}{c}\text { HLAA2+ } \\
n=33\end{array}$ & $\begin{array}{c}\text { HLAA2- } \\
n=25\end{array}$ & p \\
\hline Age & $\begin{array}{c}65.85 \\
(32-82)\end{array}$ & $\begin{array}{c}65.95 \\
(52-89)\end{array}$ & 0.863 \\
\hline Gender M/F & $21 / 12$ & $12 / 13$ & 0.234 \\
\hline T1 & 0 & 0 & $>0.05^{*}$ \\
\hline T2 & 5 & 4 & 0.930 \\
\hline T3 & 27 & 12 & 0.007 \\
\hline T4 & 1 & 9 & 0.001 \\
\hline N- & 15 & 10 & 0.678 \\
\hline $\mathrm{N}+$ & 18 & 15 & 0.678 \\
\hline MO & 28 & 18 & 0.232 \\
\hline M1 & 5 & 7 & 0.232 \\
\hline Stage I & 4 & 3 & 0.989 \\
\hline Stage II & 11 & 7 & 0.664 \\
\hline Stage III & 13 & 8 & 0.562 \\
\hline Stage IV & 5 & 7 & 0.232 \\
\hline Left colon & 20 & 7 & 0.014 \\
\hline Rectum & 13 & 18 & 0.014 \\
\hline G1 & 10 & 7 & 0.847 \\
\hline $\mathrm{G} 2+\mathrm{G} 3$ & 23 & 18 & 0.847 \\
\hline
\end{tabular}

The groups of patients with the presence or absence of HLA-A2 had similar structures in terms of age, gender, grade of differentiation (G), and TNM, and differed significantly only in terms of their T3 and T4 characteristics and location (left colon/rectum)

The analysis of clinicopathological features showed that in the group of patients with T3 tumors, patients with HLA-A2 expression predominated, while in the group of patients with T4 tumors most patients were HLA-A2-negative.

Among the patients with rectal tumors, the HLA-A2-negative phenotype dominated, while among patients with left-sided colon tumors, significantly more patients had HLA-A2 expression.

All the patients were followed-up for at least five years.

The 5-year survival rate for HLA-A2-positive patients in all stages was $72.7 \%$, while for HLA-A2-negative patients it was $40 \%$ (fig. 1).

The Kaplan Meier plot (fig. 1) showed that in the entire study group HLA-A2-positive patients lived longer then 


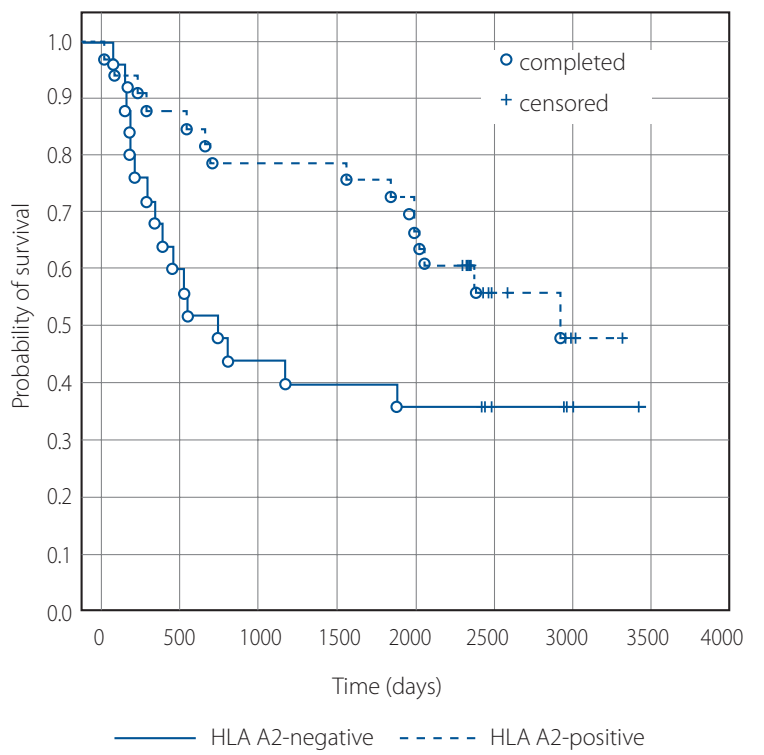

Figure 1. Cumulative proportion of survival for left-sided colorectal cancer patients in all tumor stages in HLAA2+ and HLAA2- groups

HLA-A2-negative ones. This difference was statistically significant $(p=0.027)$ according to the Wilcoxon-Gehan test.

Therefore, in our patient cohort, we found that the expression of HLA-A2 was associated with prolonged survival. In a group consisting of stage II and III CRC analyzed together, the 5-year survival rates were $75 \%$ and 36\% for HLA-A2-positive and HLA-A2-negative patients respectively. The difference between the groups however was not statistically significant (fig. 2).

An analysis of the prognostic factors in locally advanced cancer was performed.

Variables with confirmed prognostic value, such as tumor stages I-III, radicality of resection, as well as HLA-A2 status were included in the Cox proportional hazard model.

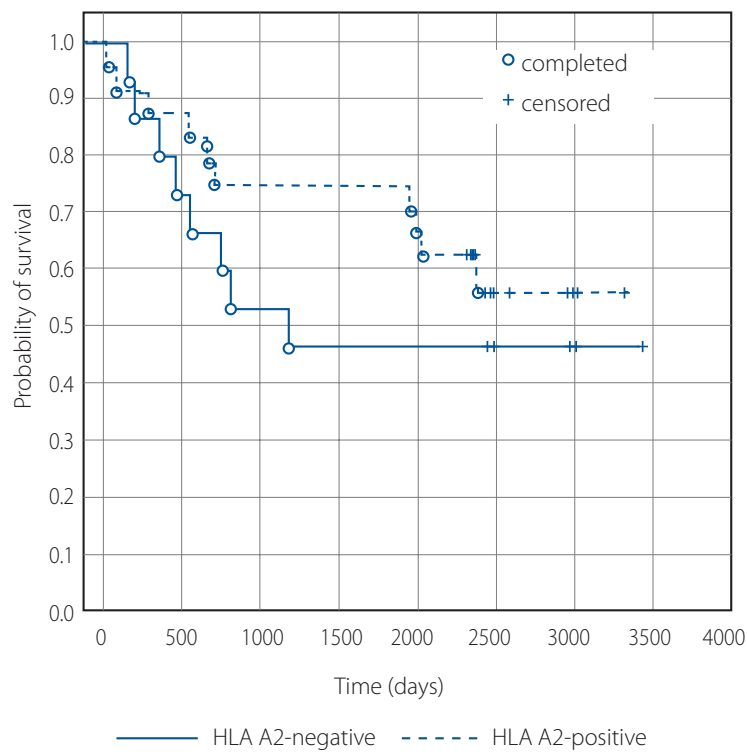

Figure 2. Cumulative proportions of survival for $\mathrm{HLA}-\mathrm{A} 2+$ and $\mathrm{HLA}-\mathrm{A} 2-$ groups of left-sided colorectal cancer patients - tumor stages II+III
HLA-A2 status was an independent prognostic factor in this group of patients ( $p=0.012 ; \mathrm{HR}=2.65$; with $95 \% \mathrm{Cl}$ for HR: 1.23-5.72). This finding showed that the lack of expression of HLA-A2 was an independent negative prognostic factor.

\section{Discussion}

The results from this study show that the presence of HLA-A2 in patients with left-sided colon and rectal cancers is an important prognostic for their outcomes. HLA-A2 is among the 8 most frequent HLA alleles (HLA $A^{*} 01, A^{*} 02, A^{*} 03, A^{*} 24, B^{*} 07$, $B^{*} 08, B^{*} 44, C^{*} 07$ ) in the Caucasian population [23]. In our study, the expression of HLA-A2 was observed in $56 \%$ patients with CRC. This percentage is slightly higher than the prevalence of HLA-A2 antigens in the Caucasian population in Central and Western Europe, which is around 45\% (42.6-51.3\%), and also higher than in the results of the study by Kiewe et al. who found a frequency of exactly 50\% of HLA-A2 expression. [2] However, these findings are not statistically significant.

The groups of patients with the presence or absence of HLA-A2 expression had similar structures in terms of age, gender, grade of differentiation (G) and TNM, and differed significantly only in terms of T3 and T4 characteristics and location (left colon, rectum). These patients were not preselected, and the researchers were not aware of their HLA status during patient recruitment.

The location of tumors does not influence survival, because, as previously mentioned, rectal and left-sided colon cancer patients have very similar prognosis. Moreover, only 3 rectal cancer patients received pre-operative radiotherapy (two HLA-A2-negative and one HLA-A2-positive). The depth of the invasion itself, the $T$ feature, is not a potent prognostic factor until the tumor cannot be radically resected. In order to overcome the imbalance in the above mentioned features, a multivariate proportional hazard model was constructed.

In our analysis, there was a significantly higher survival rate for HLA-A2-positive patients, with 72.7\% 5-year survival for the HLA-A2-positive patients and 40\% for HLA-A2-negative patients. The difference between these groups was found to be statistically significant ( $p=0.027$ ).

Our results conflict with the study by Kiewe et al. who found no statistically significant difference in 5-year survival and overall survival between HLA-A2-positive and HLA-A2-negative groups of patients with colorectal cancer [2]. One possible explanation for these differences might be differences in the patient cohort.

In the group of patients with locally advanced cancer after radical surgery, the lack of expression of HLA-A2 was an independent prognostic factor which negatively affected survival ( $p=0.012 ; H R=2.65$; with 95\% Cl for HR: 1.23-5.72). This phenomenon is not observed in all cancers. In breast cancer, HLA positive status is a favorable prognostic factor, however, in ovarian cancer it is a negative prognostic factor [7].

The 2015 Consensus of Molecular Subtypes (CMS) is considered the most robust classification system currently available 
for CRC - with clear biological interpretability - and a basis for future clinical stratification and subtype-based targeted interventions. This study identified 4 consensus molecular subtypes: CMS1 (MSI-immune), CMS2 (canonical), CMS3 (metabolic), CMS4 (mesenchymal) [24]. In relation to the anatomical location of CRC, CMS1 dominates in the right colon, CMS2 in the left colon, and CMS3 and CMS4 tumors do not have a specific anatomic location.

The group of patients in our investigation was more homogenous than in other studies, as only left-sided colon and rectal cancer patients were included. Earlier studies analyzed a mixed population of colorectal [2,25,26], colon [27], or only rectal cancer patients $[15,28]$. The authors of previously published papers did not take into account the fact that colorectal cancer may differ in biology, and thus the prognosis and response to treatment may vary, depending on the location of the tumor [18-21].

HLA-A2 expression is investigated in patients in several ways. One of the approaches uses the identification of HLA-A2 on the surface of cancer cells, other approaches identify its expression on somatic cells.

In publications, HLA status is mostly characterized in terms of the tumor environment, and its involvement in the evasion of the immune response, by analysis of its expression by the immunohistochemistry in tumor-infiltrating immune cells or in tumor cells. On the other hand, HLA status is detected in host peripheral blood cells, reflecting its role in the recognition of tumor antigens, by identifying the HLA protein using techniques such as immunoassays, flow cytometry etc., or the gene alleles, mainly by polymerase chain reaction (PCR) [10].

The interpretation and comparison of studies examining HLA class I antigen expression are generally very difficult, because the methods used for the analysis of HLA class I antigen expression vary substantially [29]. According to a recently published study, exposure to an inflammatory environment might be responsible for upregulating HLA class I gene expression in tumor cells, but the presence of HLA class I molecules at the cell surface is precluded by defects in other components of the antigen processing machinery. Besides, RNA expression analysis can detect HLA class I not only in tumors but also in immune and other stromal cells expressing this HLA. Therefore, HLA class I phenotypes should be supported by genetic data confirming mechanistic defects, while RNA expression level appears insufficient to determine HLA class I tumor status [30].

Although there are some studies assessing HLA-A on CRC tumor surface in the available literature [15, 25-28, 30], HLA-A2 expression on somatic cells in patients with CRC has not been extensively studied in the past [2].

In this study we decided to determine HLA-A2 expression on somatic cells from the peripheral blood, and therefore, it is very difficult to compare our findings with those of other studies. It would be interesting to assess HLA-A2 expression on somatic and cancer cells simultaneously, but unfortunately this was not possible due to organizational and financial constraints.
Tumor cells do not present distinctly different HLA class I antigens than the host cell, however, it should be noted that one mechanism of cancer escape from the control of the immune system is the loss, or reduction, of the expression of HLA class I antigens on cancer cell surfaces. Therefore, tumor cells may differ from somatic cells in this respect. The loss of HLA class I is rather rare (16-20\%) among MMR-p (MSS) tumors which dominate in the left half of the colon, which was confirmed on a larger cohort by ljsselsteijn et al. [30]. The conclusion from their study, that HLA class I is an important determinant of metastatic homing in CRCs, is in-line with our observation of better prognosis for patients with left-sided colorectal cancer and HLA-A2 expression.

Recently, Löffler et al. [23] provided comprehensive data on the HLA presented antigenic repertoire of CRC cells. They identified a set of 758 HLA class I and 310 HLA class II presented peptides (ligandome), exclusively expressed on colorectal carcinoma tissue, and proposed 12 naturally presented HLA class I ligands out of 38 preselected peptides (five peptides each were selected for the seven most frequent HLA allotypes in the Caucasian population and three additional peptides were selected for $\mathrm{HLA}-\mathrm{C}^{*} 07$ ), as putative candidates for future anti-CRC vaccination. Although among them only one HLA-A2 was presented, one cannot exclude the possibility that other non-selected peptides, including those related to HLA-A2, may be effective in the induction of an adaptive anti-tumor immune response, thus increasing the survival of HLA-A2 positive patients. Still more translational studies should be performed in order to understand CRC and immune system interactions.

\section{Conclusions}

In summary, the results of our study show that:

1. Patients with left-sided colorectal cancer and HLA-A2 expression lived statistically longer than HLA-A2-negative patients.

2. Lack of HLA-A2 expression was a negative prognostic factor in the group of patients with radical resections without metastases.

3. HLA-A2 status may affect the clinical course of patients with left-sided colon and rectal cancer, even though these tumors are considered less immunogenic than right-sided cancers.

The question remains whether we should consider the status of HLA-A2 expression when qualifying patients for adjuvant treatment and choosing between more or less aggressive therapies to improve their treatment results.

Funding. This research was funded by Ministry of Science and Higher Education of Poland Grants 2P05C 00129 and KV PBW/000421.

\section{Abbreviations}

APC - antigen presenting cell 


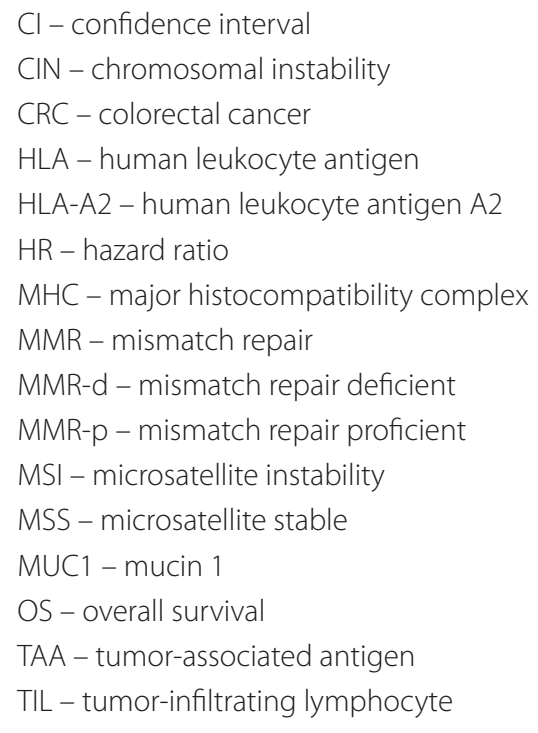

\section{Conflict of interest: none declared}

\section{Antoni M. Szczepanik}

Jagiellonian University Medical College

First Department of General, Oncological and Gastroenterological Surgery

ul. Jakubowskiego 2

31-501 Kraków, Poland

e-mail: antoni.szczepanik@uj.edu.pl

Received: $17 \mathrm{Feb} 2020$

Accepted: 20 Apr 2020

\section{References}

1. Pandolfi F, Boyle LA, Trentin L, et al. Expression of HLA-A2 antigen in human melanoma cell lines and its role in T-cell recognition. Cancer Res. 1991; 51(12): 3164-3170, indexed in Pubmed: 1904004.

2. Kiewe $P$, Mansmann V , Scheibenbogen $C$, et al. HLA-A2 expression, stage, and survival in colorectal cancer. Int J Colorectal Dis. 2008; 23(8): 767-772, doi: 10.1007/s00384-008-0488-y, indexed in Pubmed: 18461337.

3. Hee Lee Ji, Park MS, Hwang JE, et al. Dendritic cell-based immunotherapy for colon cancer using an HLA-A*0201-restricted cytotoxic T-lymphocyte epitope from tumor-associated antigen 90K. Cell Mol Immunol. 2013; 10(3): 275-282, doi: 10.1038/cmi.2012.74, indexed in Pubmed: 23524651.

4. Ferris RL, Hunt JL, Ferrone S. Human leukocyte antigen (HLA) class I defects in head and neck cancer: molecular mechanisms and clinical significance. Immunol Res. 2005; 33(2): 113-133, doi: 10.1385/IR:33:2:113, indexed in Pubmed: 16234579.

5. Madjd Z, Spendlove I, Pinder SE, et al. Total loss of MHC class I is an independent indicator of good prognosis in breast cancer. Int J Cancer. 2005; 117(2): 248-255, doi: 10.1002/ijc.21163, indexed in Pubmed: 15900607.

6. Vitale M, Pelusi G, Taroni B, et al. HLA class I antigen down-regulation in primary ovary carcinoma lesions: association with disease stage. Clin Cancer Res. 2005; 11(1): 67-72, indexed in Pubmed: 15671529.

7. Gamzatova Z, Villabona L, Dahlgren L, et al. Human leucocyte antigen (HLA) A2 as a negative clinical prognostic factor in patients with advanced ovarian cancer. Gynecol Oncol. 2006; 103(1): 145-150, doi: 10.1016/j.ygyno.2006.02.004, indexed in Pubmed: 16542716.

8. De Petris L, Bergfeldt K, Hising C, et al. Correlation between HLA-A2 gene frequency, latitude, ovarian and prostate cancer mortality rates. Med Oncol. 2004; 21(1): 49-52, doi: 10.1385/MO:21:1:49, indexed in Pubmed: 15034213

9. Ogino $\mathrm{T}$, Shigyo $\mathrm{H}$, Ishii $\mathrm{H}$, et al. HLA class I antigen down-regulation in primary laryngeal squamous cell carcinoma lesions as a poor prognostic marker. Cancer Res. 2006; 66(18): 9281-9289, doi: 10.1158/0008-5472. CAN-06-0488, indexed in Pubmed: 16982773.
10. Mezquita L, Charrier M, Faivre L, et al. Prognostic value of HLA-A2 status in advanced non-small cell lung cancer patients. Lung Cancer. 2017; 112: 10-15, doi: 10.1016/j.lungcan.2017.07.004, indexed in Pubmed: 29191581.

11. Dierssen JW, de Miranda NF, Mulder A, et al. High-resolution analysis of HLA class I alterations in colorectal cancer. BMC Cancer. 2006; 6: 233, doi: 10.1186/1471-2407-6-233, indexed in Pubmed: 17014712.

12. Kloor $M$, Becker $C$, Benner A, et al. Immunoselective pressure and human leukocyte antigen class I antigen machinery defects in microsatellite unstable colorectal cancers. Cancer Res. 2005; 65(14): 6418-6424, doi: 10.1158/0008-5472.CAN-05-0044, indexed in Pubmed: 16024646.

13. Söreide $K$, Janssen EAM, Söiland $H$, et al. Microsatellite instability in colorectal cancer. Br J Surg. 2006; 93(4): 395-406, doi: 10.1002/bjs.5328, indexed in Pubmed: 16555243.

14. Kim K, Castro EJ, Shim H, et al. Differences Regarding the Molecular Features and Gut Microbiota Between Right and Left Colon Cancer. Ann Coloproctol. 2018; 34(6): 280-285, doi: 10.3393/ac.2018.12.17, indexed in Pubmed: 30630301.

15. Speetjens FM, de Bruin EC, Morreau H, et al. Clinical impact of HLA class I expression in rectal cancer. Cancer Immunol Immunother. 2008; 57(5): 601-609, doi: 10.1007/s00262-007-0396-y, indexed in Pubmed: 17874100 .

16. Passardi A, Canale M, Valgiusti M, et al. Immune Checkpoints as a Target for Colorectal Cancer Treatment. Int J Mol Sci. 2017; 18(6), doi: 10.3390/ ijms18061324, indexed in Pubmed: 28635639.

17. Wang RF, Wang HY. Immune targets and neoantigens for cancer immunotherapy and precision medicine. Cell Res. 2017; 27(1): 11-37, doi: 10.1038/cr.2016.155, indexed in Pubmed: 28025978.

18. Lim DRo, KukJK, Kim T, et al. Comparison of oncological outcomes of right-sided colon cancer versus left-sided colon cancer after curative resection: Which side is better outcome? Medicine (Baltimore). 2017; 96(42): e8241, doi: 10.1097/MD.0000000000008241, indexed in Pubmed: 29049212.

19. Benedix F, Kube R, Meyer F, et al. Colon/Rectum Carcinomas (Primary Tumor) Study Group. Comparison of 17,641 patients with right- and left-sided colon cancer: differences in epidemiology, perioperative course, histology, and survival. Dis Colon Rectum. 2010; 53(1): 57-64, doi: 10.1007/DCR.0b013e3181c703a4, indexed in Pubmed: 20010352.

20. Derwinger K, Gustavsson B. Variations in demography and prognosis by colon cancer location. Anticancer Res. 2011;31(6): 2347-2350, indexed in Pubmed: 21737663.

21. Shen $\mathrm{H}$, Yang J, Huang $\mathrm{Q}$, et al. Different treatment strategies and molecular features between right-sided and left-sided colon cancers. World J Gastroenterol. 2015; 21(21): 6470-6478, doi: 10.3748/wjg.v21. i21.6470, indexed in Pubmed: 26074686.

22. de Vries NL, Swets M, Vahrmeijer AL, et al. The Immunogenicity of Colorectal Cancer in Relation to Tumor Development and Treatment. Int J Mol Sci. 2016; 17(7), doi: 10.3390/ijms17071030, indexed in Pubmed: 27367680.

23. Löffler MW, Kowalewski DJ, Backert L, et al. Mapping the HLA Ligandome of Colorectal Cancer Reveals an Imprint of Malignant Cell Transformation. Cancer Res. 2018; 78(16): 4627-4641, doi: 10.1158/0008-5472. CAN-17-1745, indexed in Pubmed: 29789417.

24. Guinney J, Dienstmann R, Wang X, et al. The consensus molecular subtypes of colorectal cancer. Nat Med. 2015; 21(11): 1350-1356, doi: 10.1038/nm.3967, indexed in Pubmed: 26457759.

25. Sandel MH, Speetjens FM, Menon AG, et al. Natural killer cells infiltrating colorectal cancer and MHC class I expression. Mol Immunol. 2005; 42(4): 541-546, doi: 10.1016/j.molimm.2004.07.039, indexed in Pubmed: 15607811.

26. Swets $M$, König $M H$, Zaalberg $A$, et al. HLA-G and classical HLA class I expression in primary colorectal cancer and associated liver metastases. Hum Immunol. 2016; 77(9): 773-779, doi: 10.1016/j.humimm.2016.03.001, indexed in Pubmed: 26968946.

27. Zeestraten ECM, Reimers MS, Saadatmand S, et al. Combined analysis of HLA class I, HLA-E and HLA-G predicts prognosis in colon cancer patients. Br J Cancer. 2014; 110(2): 459-468, doi: 10.1038/bjc.2013.696, indexed in Pubmed: 24196788.

28. Reimers MS, Engels CC, Putter $\mathrm{H}$, et al. Prognostic value of HLA class I, HLA-E, HLA-G and Tregs in rectal cancer: a retrospective cohort study. BMC Cancer. 2014; 14: 486, doi: 10.1186/1471-2407-14-486, indexed in Pubmed: 24997850.

29. Kloor M, Michel S, von Knebel Doeberitz M. Immune evasion of microsatellite unstable colorectal cancers. Int J Cancer. 2010; 127(5): 1001-1010, doi: 10.1002/ijc.25283, indexed in Pubmed: 20198617.

30. Ijsselsteijn ME, Petitprez F, Lacroix $L$, et al. Revisiting immune escape in colorectal cancer in the era of immunotherapy. Br J Cancer. 2019; 120(8): 815-818, doi: 10.1038/s41416-019-0421-x, indexed in Pubmed:30862951. 